\title{
Vascular smooth muscle cell phenotypes in primary pulmonary hypertension
}

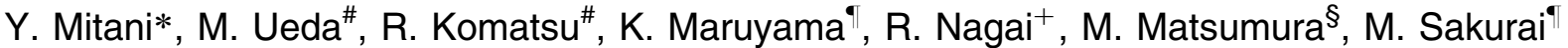

\begin{abstract}
Vascular smooth muscle cell phenotypes in primary pulmonary hypertension. Y. Mitani, M. Ueda, R. Komatsu, K. Maruyama, R. Nagai, M. Matsumura, M. Sakurai. (C)ERS Journals Ltd 2001.

ABSTRACT: Primary pulmonary hypertension (PPH) is associated with specific structural alterations, including cellular intimal thickening, intimal fibrosis, and plexiform lesions.

To determine the phenotypes of smooth muscle cells (SMCs) in such lesions, the authors conducted an immunohistochemical analysis of lung tissues from two patients with PPH, using two antimuscle actin antibodies, HHF35 and CGA7, and two antiSMC myosin heavy chain markers, anti-SM1 and anti-SM2 antibodies and related antibodies. Cells that stained positive $(+)$ with HHF35, CGA7, anti-SM1, and antiSM2 were considered to be SMCs of a mature state. Conversely, those that stained positive with HHF35 and anti-SM1, but weakly positive $(+I-)$ or negative $(-)$ with CGA7 and anti-SM2, were considered to be SMCs exhibiting an immature state.

Cellular intimal thickening was composed of SMCs of an immature phenotype (HHF35+, CGA7+I-, SM1 +, SM2+l-), accompanied by the expression of fibronectin and the presence of macrophages; intimal fibrosis contained mature SMCs (HHF35+, CGA7+, SM1 +, SM2+); and plexiform lesion consisted of proliferative endothelial cells (von Willebrand factor-positive cells, proliferating cell nuclear antigen-positive cells) and underlying immature SMCs (HHF35 +, CGA7-, SM1 +, SM2-) associated with fibronectin expression and macrophage infiltration.

These findings suggest that smooth muscle cells with specific phenotypes may contribute to the development of specific vascular lesions in primary pulmonary hypertension.
\end{abstract}

Eur Respir J 2001; 17: 316-320.

\begin{abstract}
*Depts of Pediatrics and ${ }^{\#}$ Anesthesiology, Mie University School of Medicine, Tsu, Mie, Japan. Dept of Pathology, Osaka City University Medical School, Abeno-ku, Osaka, Japan. ${ }^{+}$Second Dept of Medicine, Gunma University School of Medicine, Maebashi, Gunma, Japan. ${ }^{8}$ Dept of Pediatrics, Tenri Hospital, Tenri, Nara, Japan.
\end{abstract}

Correspondence: M. Ueda, Dept of Pathology, Osaka City University Medical School, 1-4-3 Asahi-machi, Abenoku, Osaka, Japan.

Fax: 81666453742

\author{
Keywords: Cytoskeletal proteins \\ fibronectin \\ intimal hyperplasia \\ macrophage \\ plexiform lesion
}

Received: January 131999

Accepted after revision July 182000
Pulmonary hypertension is associated with specific structural alterations that progress from medial thickening to cellular intimal thickening, intimal fibrosis, and plexiform lesions [1]. Although early reports have shown that the vascular smooth muscle cells (SMCs) are contained in hypertensive pulmonary vascular lesions [2,3], the phenotype of SMCs in these specific lesions has not been well determined. Using a series of new actin and myosin heavy chain markers, the phenotypes of SMCs in the neointima following percutaneous transluminal coronary angioplasty (PTCA) have recently been demonstrated as well as in atheromatous plaques [4-6]. In this study, to determine the phenotypes of SMCs in pulmonary vascular lesions associated with primary pulmonary hypertension (PPH), an immunohistochemical analysis of lung tissues from patients with PPH was conducted, using a variety of antismooth muscle (anti-SM) and related antibodies.

\section{Methods}

Lung tissues were obtained at autopsy from two patients with PPH, an 8-yr-old boy and a 28 -yr-old male. The 8-yr-old male was referred to hospital because of an episode of fainting and oedema. At that time, he was in the terminal stage of the disease, as indicated by cardiomegaly (cardiothoracic ratio; 0.65 ) in chest radiography and suprasystemic pulmonary arterial pressure in ultrasound examination. These findings, together with those in magnetic resonance imaging and lung perfusion scan, revealed no other causes of pulmonary hypertension. The patient had progressive heart failure regardless of medication and died 6 months later. The 28 -yr-old male had been fairly stable under observation for $10 \mathrm{yrs}$ after an initial episode of haemoptysis. Cardiac catheterization was performed 7 months before he died of heart failure, and pulmonary-to-aortic systemic pressure ratio was 0.83 with no pulmonary vascular response to inhaled nitric oxide. No other causes of pulmonary hypertension were revealed. Lung tissues, obtained at autopsy from three patients without pulmonary hypertension (21-yr-old male with malignant lymphoma; 29-yr-old male with embryonal carcinoma; 38-yr-old male with fulminant hepatitis), were also studied as controls.

The tissues were fixed in methanol-Carnoy's solution and embedded in paraffin. Twenty serial sections from 

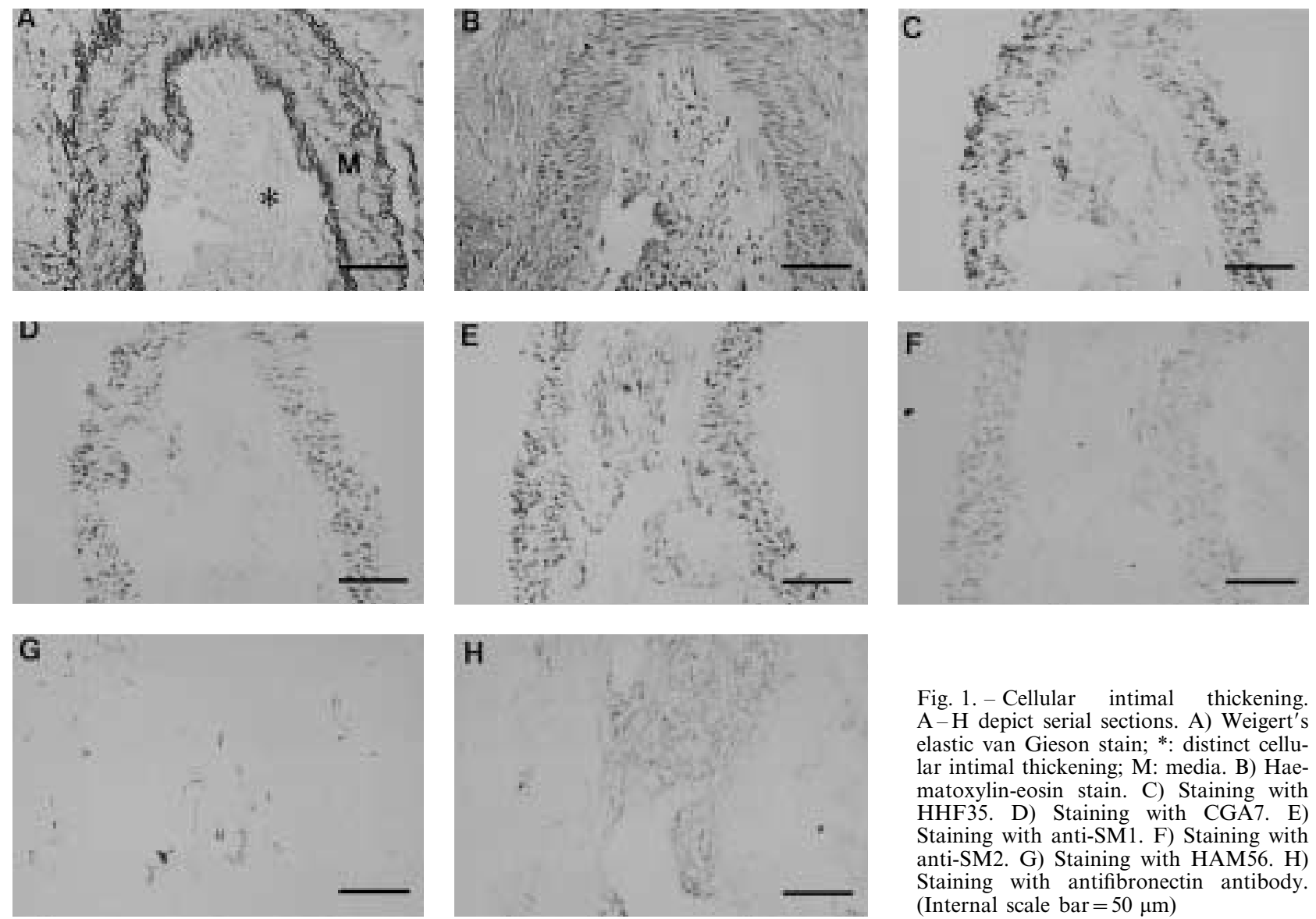

Fig. 1. - Cellular intimal thickening. A $-\mathrm{H}$ depict serial sections. A) Weigert's elastic van Gieson stain; *: distinct cellular intimal thickening; M: media. B) Haematoxylin-eosin stain. C) Staining with HHF35. D) Staining with CGA7. E) Staining with anti-SM1. F) Staining with anti-SM2. G) Staining with HAM56. H) Staining with antifibronectin antibody. (Internal scale bar $=50 \mu \mathrm{m}$ )

each block were cut $5 \mu \mathrm{m}$ thick. Every first and second section was stained with haematoxylin-eosin and Weigert's elastic van Gieson stain, respectively. The remaining sections were used for immunohistochemical staining. The labelled streptavidin-biotin complex system with nickel chloride colour modification was employed. Sections were counter stained with methyl green. SMCs in the vascular lesions were immunophenotyped using two antimuscle actin markers, HHF35 (Dako A/S, Glostrup, Denmark) and CGA7 (Enzo Biochem, Inc, New York, NY), and two anti-SMC myosin heavy chain markers, anti-SM1 and anti-SM2 antibodies, as reported in our studies on the immunophenotypic modulation of SMC in the neointima at the site of PTCA and in atheromatous plaques in humans [4-6]. The anti-SM1 and anti-SM2 antibodies (antiSM1 and anti-SM2, respectively) recognize two myosin heavy chain isoforms of SMCs, SM1 and SM2, respectively, which are highly specific markers for SMC lineage [5, 6]. Immunohistochemical findings using HHF35, CGA7, anti-SM1, anti-SM2, and antifibronectin antibody were analysed by two of the investigators ( $\mathrm{YM}$ and $\mathrm{MU}$ ) in a semiquantitative manner as follows: ++ , more than two-thirds of cells positive; + , about half of cells positive; $+/-,<10 \%$ positive; -, negative. Cells that stained positive with HHF35, CGA7, anti-SM1, and anti-SM2 were considered to be SMC of a mature state; conversely, those that stained positive with HHF35 and anti-SM1, but weakly positive or negative with CGA7 and anti-SM2 were considered to be phenotypically modulated SMC exhibiting an immature state [4-6]. The monoclonal antibody against proliferating cell nuclear antigen (PCNA; Dako A/S) was used to detect proliferating cells. The other monoclonal antibodies used were antifibronectin antibody (Cosmo Bio Co. Ltd., Tokyo, Japan); antimacrophage antibody, HAM56 (Dako A/S); and antivon Willebrand factor (vWf) antibody (Dako A/S).

\section{Results}

Cellular intimal thickening, intimal fibrosis and plexiform lesions were observed in the lung tissue of both patients with PPH (Heath-Edwards classification Grade 4). No intimal lesions were found in the lung tissue of control patients. Cellular intimal thickening contained cells that exhibited oval nuclei and copious cytoplasm with scanty deposition of elastin (fig. 1A and B). Cells in these lesions stained positive with HHF35 and anti-SM1, but only weakly with CGA7 and antiSM2 (fig. 1C-F; table 1). These lesions revealed a marked amount of fibronectin and a distinct accumulation of macrophages (fig. $1 \mathrm{G}$ and $\mathrm{H}$; table 1). PCNApositive cells were also found in the intima. Intimal fibrosis contained spindle-shaped cells, and exhibited dense deposition of elastin (fig. 2A). Cells in these 
Table 1.-Results of the immunohistochemical studies

\begin{tabular}{|c|c|c|c|c|c|}
\hline & \multicolumn{4}{|c|}{ SMC phenotype } & \multirow[b]{3}{*}{ Fibronectin } \\
\hline & \multicolumn{2}{|c|}{ Actin markers } & \multicolumn{2}{|c|}{ MHC markers } & \\
\hline & HHF-35 & CGA7 & anti-SM1 & anti-SM2 & \\
\hline Cellular intimal thickening & ++ & $+/-$ & ++ & $+/-$ & ++ \\
\hline Intimal fibrosis & ++ & ++ & ++ & ++ & - \\
\hline Plexiform lesion & ++ & - & ++ & - & + \\
\hline Media & ++ & ++ & ++ & ++ & - \\
\hline
\end{tabular}

SMC: smooth muscle cell; MHC: myosin heavy chain; ++ : more than two-thirds of cells positive; + : half of cells positive; $+/-:<10 \%$ of cells positive; -: negative.

lesions stained positive with HHF35, CGA7, anti-SM1, and anti-SM2 (fig. 2C and D; table 1). These lesions showed a small amount of fibronectin (fig. 2B; table 1), with only few scattered macrophages. PCNA-positive cells were absent. The media in pulmonary vessels of two patients with PPH and three control patients was homogeneous and stained positive with HHF35, CGA7, anti-SM1, and anti-SM2, and showed neither fibronectin expression nor PCNA positivity (table 1).

Plexiform lesions contained a plexus of aneurysmal and angiomatoid small vessels (fig. 3A). von Willebrand factor (vWf)-positive endothelial cells were found on the luminal surface in the lesion (fig. 3B). Cells below the endothelial cells stained positive with HHF35 and anti-SM1, but negative with CGA7 and anti-SM2 (fig. 3C and D; table 1). The lesions exhibited a distinct
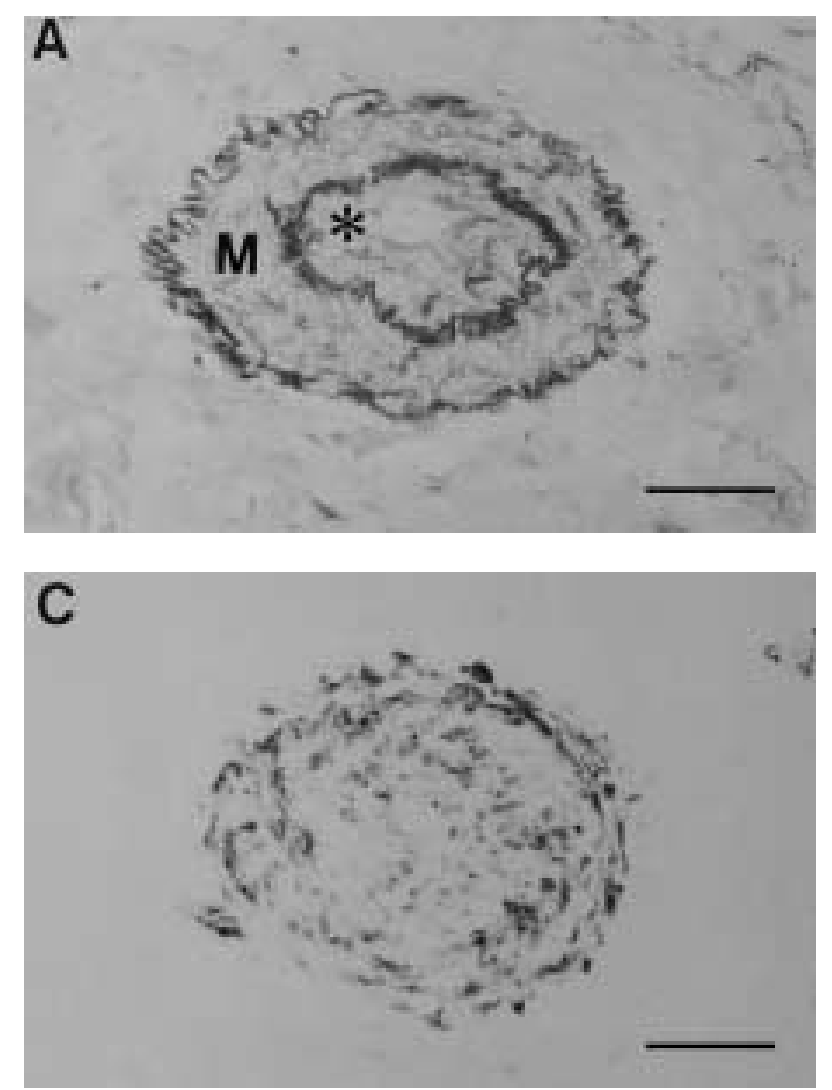

Fig. 2. - Intimal fibrosis. Panels A-D depict serial sections. A) Weigert's elastic van Gieson stain; *: intimal fibrosis; M: media. B) Staining with antifibronectin antibody. C) Staining with anti-SM1. D) Staining with anti-SM2. (Internal scale bar=30 $\mu$ m).
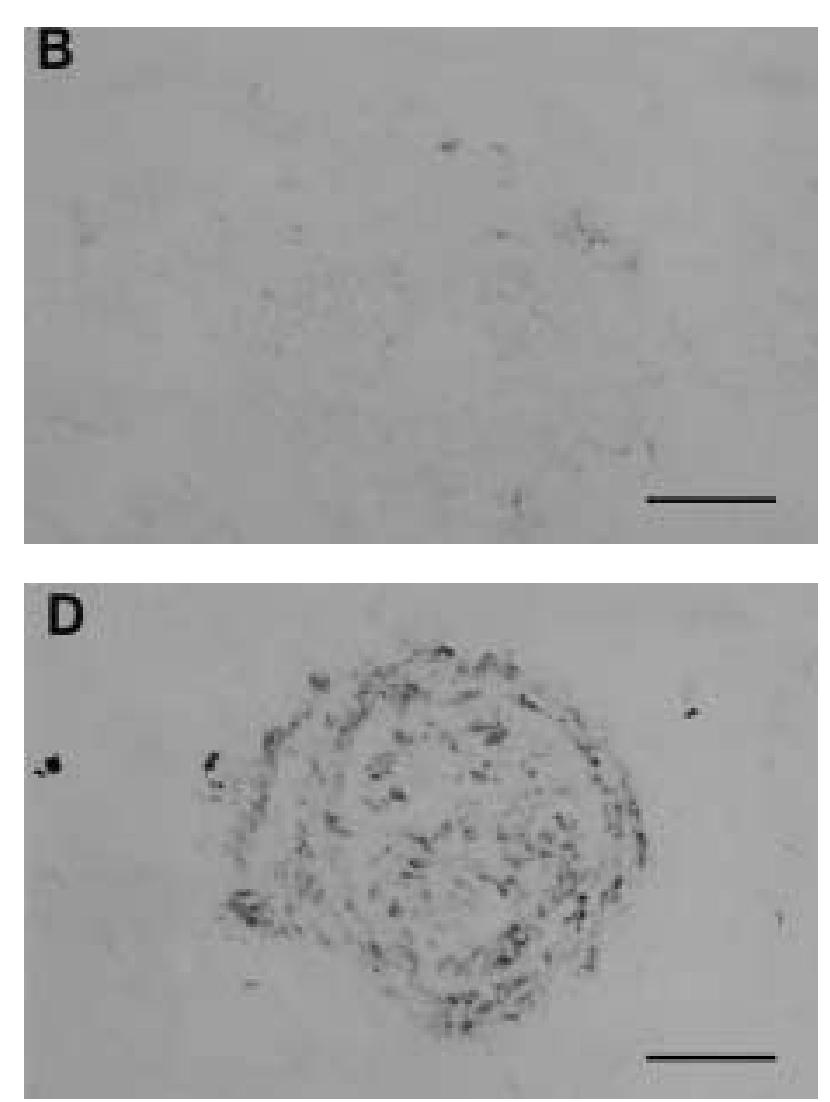

expression of fibronectin (fig. 3E; table 1) and the infiltration of macrophages. In addition, PCNA positivity was observed in endothelial cells and the cells adjacent to the luminal surface (fig. $3 \mathrm{~F}$ ).

\section{Discussion}

The major findings in the lung tissues of the two patients with PPH were as follows: a) cellular intimal thickening was composed of SMCs of an immature phenotype $(\mathrm{HHF} 35++, \mathrm{CGA} 7 \pm$, SM1 ++ , SM2 \pm ), accompanied by the expression of fibronectin $(++)$ and the presence of macrophages; b) intimal fibrosis contained SMCs of a mature phenotype $(\mathrm{HHF} 35++, \mathrm{CGA} 7++, \mathrm{SM} 1++, \mathrm{SM} 2++)$; 

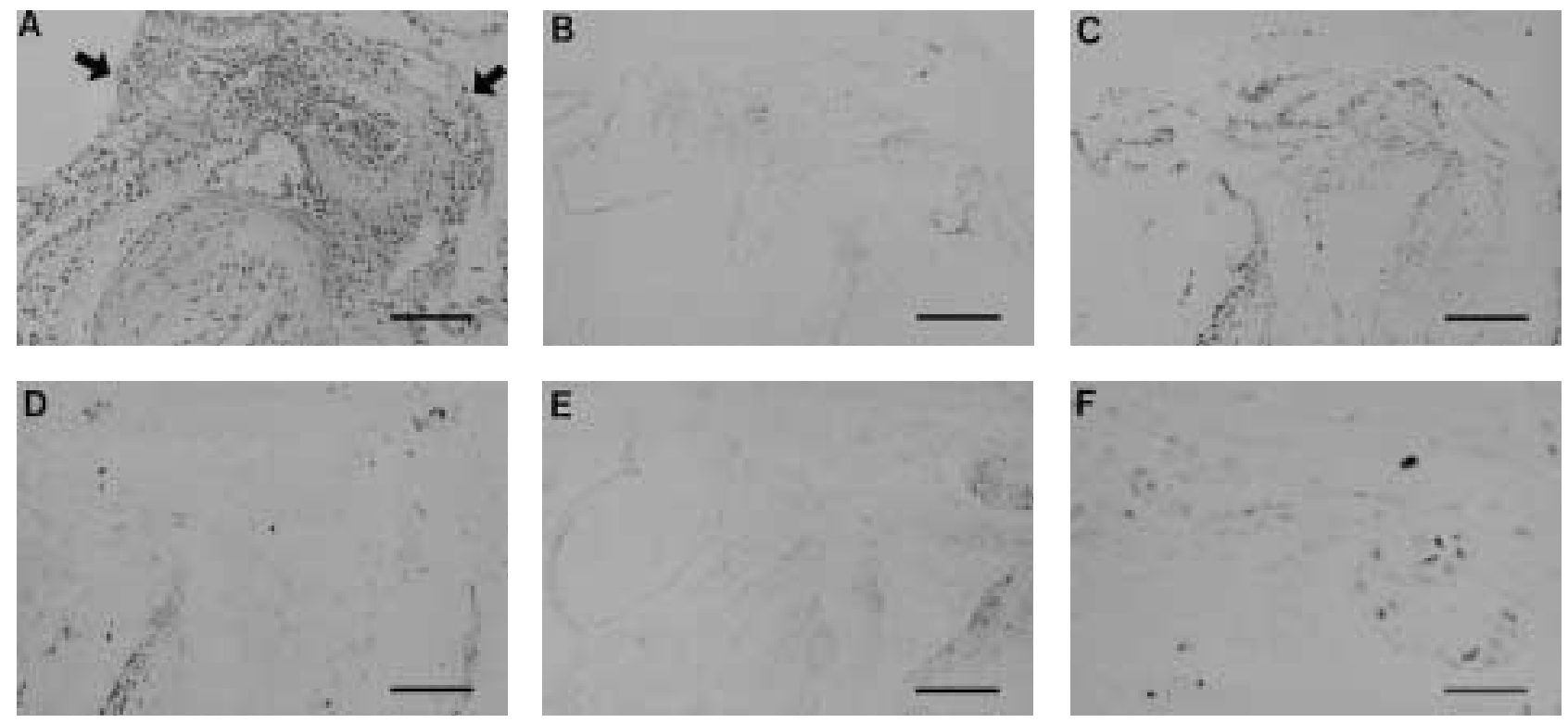

Fig. 3. - Plexiform lesions. Panels A-F depict serial sections. A) Haematoxylin-eosin stain. Plexiform lesions (arrows) are seen. B) Staining with anti-von Willebrand factor antibody. C) Staining with anti-SM1. D) Staining with anti-SM2. E) Staining with antifibronectin antibody. F) Staining with anti-PCNA antibody. (Internal scale bars: $\mathrm{A}=80 \mu \mathrm{m} ; \mathrm{B}-\mathrm{E}=60 \mu \mathrm{m} ; \mathrm{F}=25 \mu \mathrm{m}$ )

and c) plexiform lesion consisted of proliferative endothelial cells (vWf-positive, PCNA-positive) and underlying immature SMCs (HHF35 + +, CGA7 -, $\mathrm{SM} 1++$, SM2 -) associated with fibronectin expression $(+)$ and macrophage infiltration. The media consisted of a mature phenotype (HHF35 ++ , CGA $7++$, SM1 ++ , SM2 ++ ) of SMC without fibronectin deposition or macrophage infiltration. These findings were obtained by the combined use of a series of new antimuscle antibodies, which distinguished the mature from the immature phenotype of SMC in atherosclerotic and balloon-injured coronary vessels [4-6].

The present study demonstrated that intimal lesions associated with PPH contained SMCs with different phenotypes. These findings are consistent with an earlier report that described the varied immunoreactivity in intimal lesions, with some being immunonegative, and others being immunopositive for $\alpha$ actin [7]. The present findings of intimal lesions are interesting, because the pnenotypic change of SMCs might be associated with the formation of specific intimal lesions in PPH. The results on neointimal lesions in PPH resemble previous findings in the neointima after PTCA in human coronary arteries $[4,5]$. It was observed that, on the 20th day after PTCA, the neointima consisted of immature SMCs that stained positive with HHF35 but negative with CGA7. After 5 months, the intimal lesion was composed of mature SMCs that stained positive with both HHF35 and CGA7 [4]. Similarly, at $2-5$ months after PTCA, the neointimal cells expressed SM1 but not SM2. After 6 months, they were positive for both SM1 and SM2, like those in the normal media [5]. These observations strongly suggest that phenotypic changes of SMCs contribute to the healing process after PTCA injury $[4,5]$. In the present study, however, the association of phenotypic changes of SMCs with the progression of intimal lesions could not be determined, since lung sections were examined only in the terminal stage of the disease.

PCNA positivity was found in the endothelium and the cells adjacent to the luminal surface of plexiform lesions, suggesting a contribution of neovascularization to lesion formation. Previous reports on the cellular composition of plexiform lesions have been controversial. Ultrastructural studies have demonstrated that plexiform lesions consist of myofibroblasts and cells of undetermined nature that contain cytoplasmic filaments, so-called fibrillary cells [3]. Conversely, a recent study that used immunocytochemical staining indicated that the plexiform lesion is an angiogenic lesion composed of proliferative endothelial cells [8]. The present study showed that the subendothelial cells stained positive with HHF35 and anti-SM1, but negative with CGA7 and anti-SM2, suggesting the presence of immature SMC in the subendothelium. These cells may correspond to the myofibroblasts, as observed in a previous ultrastructural study [3]. These observations suggest that neovascularization accompanied by the appearance of underlying immature SMCs may contribute to the formation of plexiform lesions.

The observation that immature SMCs with proliferative activity were found together with macrophages and fibronectin expression in intimal hyperplasia and plexiform lesions may suggest an active role of the immature SMCs in the pathogenesis of these lesions. A previous report demonstrated that the neointimal macrophages in human PPH are found together with fibronectin expression, which may be induced by cytokines released by the macrophages [9]. Infiltration by macrophages is common in the plexiform lesions in human PPH, suggesting that cytokines released by macrophages may affect the development of such lesions [8]. An in vitro study indicates that fibronectin promotes the modulation of SMCs from a contractile to a synthetic phenotype [10]. Taken together, these findings suggest 
that the presence of infiltrating macrophages and the expression of fibronectin may be important in the morphogenesis of intimal and plexiform lesions of PPH via the phenotypic modulation of SMCs.

In summary, the preliminary study demonstrated that cellular intimal thickening and plexiform lesions contained immature smooth muscle cells, which were associated with the findings suggesting active vascular remodelling: proliferating cell nuclear antigen positivity of smooth muscle cells, macrophage infiltration and fibronectin deposition. In contrast, intimal fibrosis contained mature smooth muscle cells without such active findings. These findings suggest that smooth muscle cells with specific phenotypes may contribute to the development of specific vascular lesions in pulmonary hypertension. Future studies with more cases are needed to further elucidate the relationship between smooth muscle cell phenotypes and specific vascular lesions in pulmonary hypertension.

\section{References}

1. Heath D, Edwards JE. The pathology of hypertensive pulmonary vascular disease: a description of six grades of structural changes in the pulmonary arteries with special references to congenital cardiac defects. Circ 1958; 18: $533-547$.

2. Heath D, Smith P, Gosney J. Ultrastructure of early plexogenic pulmonary arteriopathy. Histopathol 1988; 12: $41-52$.
3. Smith P, Heath D. Electron microscopy of the plexiform lesion. Thorax 1979; 34: 177-186.

4. Ueda M, Becker AE, Tsukada T, Numano F, Fujimoto T. Fibrocellular tissue response after percutaneous transluminal coronary angioplasty. Circ 1991; 83: $1327-1332$.

5. Aikawa M, Sakomura $\mathrm{Y}$, Ueda $\mathrm{M}$, et al. Redifferentiation of smooth muscle cells after coronary angioplasty determined via myosin heavy chain expression. Circ 1997; 96: 82-90.

6. Aikawa M, Silvam PN, Kuro-o M, et al. Human smooth muscle myosin heavy chain isoforms as molecular markers for vascular development and atherosclerosis. Circ Res 1993; 73: 1000-1012.

7. Allen K, Haworth SG. Cytoskeletal features of immature pulmonary vasculature smooth muscle cells: The influence of pulmonary hypertension on normal development. J Pathol 1989; 158: 311 317.

8. Tuder RM, Groves B, Badesch DB, Voelkel NF. Exuberant endothelial cell growth and elements of inflammation are present in plexiform lesions of pulmonary hypertension. Am J Pathol 1994; 144: $275-285$.

9. Liptay MJ, Parks WC, Mecham RP, et al. Neointimal macrophages colocalize with extracellular matrix gene expression in human atherosclerotic pulmonary arteries. J Clin Invest 1993; 91: 588-594.

10. Thyberg J, Heiden Y, Sjolung M, Palmberg L, Bottger BA. Regulation of differentiated properties and proliferation of arterial smooth muscle cells. Arteriosclerosis 1990; 10: 966-990. 